\title{
Neoadjuvant nimotuzumab plus chemoradiotherapy compared to neoadjuvant chemoradiotherapy and neoadjuvant chemotherapy for locally advanced esophageal squamous cell carcinoma This article has an addendum: Oncotarget. 2022; 13:1300-1300.
}

\author{
Yongshun Chen ${ }^{1,2}$, Xiaoyuan $\mathrm{Wu}^{2}$, Daxuan $\mathrm{Hao}^{2}$, Xinyu Cheng ${ }^{2}$, Lei Zhang ${ }^{3}$, Yougai \\ Zhang $^{2}$, Shaobo Ke ${ }^{1}$, Wei Shi ${ }^{1}$ and Chunyu $\mathrm{He}^{2}$ \\ ${ }^{1}$ Department of Clinical Oncology, Renmin Hospital of Wuhan University, Wuhan, China \\ ${ }^{2}$ Department of Radiation Oncology, Zhengzhou University Affiliated Cancer Hospital, Zhengzhou, China \\ ${ }^{3}$ Department of Nephrology, People's Hospital of Tibet Autonomous Region, Tibet Autonomous Region, Lhasa, China \\ Correspondence to: Yongshun Chen, email: yongshun2007@163.com \\ Keywords: esophageal cancer; locally advanced disease; neoadjuvant therapy; EGFR-inhibitor; pathological complete \\ response
}

Received: August 14, 2017 Accepted: October 29, 2017 Epub: January 03, 2018 Published: June 18, 2019

Copyright: Chen et al. This is an open-access article distributed under the terms of the Creative Commons Attribution License 3.0 (CC BY 3.0), which permits unrestricted use, distribution, and reproduction in any medium, provided the original author and source are credited.

\section{ABSTRACT}

Neoadjuvant therapy improves long-term locoregional control and overall survival after surgical resection for esophageal cancer, and neoadjuvant chemotherapy (nCT) or neoadjuvant chemoradiotherapy (nCRT) are commonly used in clinical practice. Nimotuzumab is a humanized monoclonal antibody against epidermal growth factor receptor (EGFR), the efficacy of nimotuzumab added to nCRT for esophageal cancer is uncertain. We conducted this retrospective study in which combining neoadjuvant treatment of nimotuzumab with chemoradiotherapy (NimonCRT) is compared with nCRT and nCT for patients with potentially resectable locally advanced esophageal squamous cell carcinoma. One hundred ninety-five patients received neoadjuvant therapy and $172(88.2 \%)$ underwent esophagectomy. Surgical resection was performed in $\mathbf{9 4 . 4 \%}$ after Nimo-nCRT, versus $92.5 \%$ after nCRT and $83.5 \%$ after $\mathrm{nCT}(P=0.026)$. The RO resection rate was $100 \%$ after Nimo-nCRT, 95.9\% after $\mathrm{nCRT}$ and $\mathbf{9 2 . 6 \%}$ after $\mathrm{nCT}(P=0.030)$. Pathological complete response (pCR) was achieved in $\mathbf{4 1 . 2 \%}$ after Nimo-nCRT, versus $32.4 \%$ after $n$ CRT and $\mathbf{1 4 . 8 \%}$ after nCT $(P=0.0001)$. Lymph-node metastases were observed in $29.4 \%$ in the Nimo-nCRT group, versus $21.6 \%$ in the nCRT group and $35.8 \%$ in the nCT group $(P=0.093)$. More patients in the Nimo-nCRT and nCRT group developed grade 3 esophagitis compared to those in the nCT group, $P=0.008$. There was no difference in surgical complications between the treatment groups. $n C R T$ results in improved RO resection, higher PCR rate, and a lower frequency of lymph node metastases compared to nCT, adding nimotuzumab to nCRT is safe and appears to facilitate complete resection and increase the PCR rate.

\section{INTRODUCTION}

Esophageal cancer is an aggressively human malignancy, surgical resection by itself provides a high degree of locoregional relapse and distant metastasis for locally advanced disease [1]. Neoadjuvant therapy improves long-term locoregional control and overall survival for esophageal cancer patients undergoing esophagectomy, and two main neoadjuvant approaches are commonly used in clinical practice. The first is neoadjuvant chemotherapy (nCT) using the $\mathrm{OEO} 2$ protocol, and demonstrated a 5-year survival improvement of $6 \%$ compared to surgery alone [1]. The other is neoadjuvant chemoradiotherapy (nCRT) based on the CROSS regimen, which showed a significant improvement in 5-year survival rate in comparison to surgery alone $(47 \%$ vs. $33 \%, P=0.003)[2,3]$.

R0 resection, pathological complete response (pCR) and downstaging have been regarded as 
strong and relevant predictors of increased survival in esophageal cancer patients who were undergoing neoadjuvant therapy $[1,4-6]$, nCRT shows the advantages of effective local therapy in combination with systemic treatment, and the benefits of the radiosensitising effect of chemotherapy compared with nCT. The recently published NeoRES trial in a mixed cohort of 181 patients with esophageal squamous cell carcinoma and adenocarcinoma of the distal esophagus, manifested that $\mathrm{nCRT}$ increases the $\mathrm{pCR}$ and $\mathrm{R} 0$ resection rates and decreases the proportion of patients with metastases in regional lymph nodes compared to $\mathrm{nCT}$, though dose not significantly improve overall survival in squamous cell carcinoma patients [7].

The epidermal growth factor receptor (EGFR) signal pathway plays an important role in the carcinogenesis and progress of esophageal cancer. EGFR expression is observed in $50-70 \%$ of esophageal cancer patients and is correlated with inferior prognosis $[8,9]$. Nimotuzumab is a recombinant humanized monoclonal IgG1 antibody against human EGFR and it can effectively block the binding of EGF and transforming growth factor-alpha to EGFR. In several phase II studies, nimotuzumab concurrently with chemotherapy and radiotherapy have been proven to be safe and effective in the treatment of esophageal cancer [10-13]. Ramos-Suzarte and colleagues [10] compared nimotuzumab plus concurrent chemoradiotherapy with 5-fluorouracil and cisplatin in the treatment of stage III/IV esophageal squamous cell carcinoma patients and resulted in a great improvement in efficacy ( 48 vs $15 \%, P=0.014$ ), the disease control rate (61 vs $27 \%, P=0.017$ ) and median overall survival (8.1 vs 3.0 months) in the nimotuzumab group. However, the safety and efficacy of the combination of nimotuzumab with neoadjuvant chemoradiotherapy (Nimo$\mathrm{nCRT}$ ) in patients with resectable esophageal squamous cell carcinoma is unclear. Therefore, we conducted this study to compare the rate of pCR after Nimo-nCRT with that after $\mathrm{nCRT}$ and after $\mathrm{nCT}$. Surgical resection rate, $\mathrm{R} 0$ resection rate, downstaging and number of lymph node metastases were also investigated.

\section{RESULTS}

\section{Patient characteristics}

In total, 195 patients with locally advanced squamous cell carcinoma of the thoracic esophagus were included between June 2010 and May 2015. The median age at enrollment was 59 years and the majority of patients were male $(n=152,77.9 \%)$. The most common sites of primary tumor were the upper $(28.4 \%)$ and middle portion $(65.1 \%)$ of the thoracic esophagus. Preoperative staging showed that $23.6 \%$ of patients were clinical stage IIA, $36.4 \%$ of patients were stage IIIA, and $33.8 \%$ of patients were stage IIIC. Clinical and demographic data for the three groups are shown in Table 1.

\section{Toxicity}

The most frequently observed hematologic grade 3 or 4 adverse event preoperatively was neutropenia, which was noted in $24.8 \%$ of patients in the nCT group, $31.3 \%$ in the nCRT group and $27.8 \%$ in the Nimo-nCRT group, respectively, no statistical significance was found among the three groups $(P=0.640)$. The incidence of febrile neutropenia was comparable in the three groups $(P=$ $0.819)$. The most frequently occurring nonhematologic grade 3 or 4 adverse events in the three groups were fatigue, anorexia, constipation, nausea, and vomiting.

Compared to those in the $\mathrm{nCT}$ group, more patients in the nCRT and in the Nimo-nCRT group developed grade 3 esophagitis $(P=0.008)$. The median time to report of esophagitis was 19 days (range: 15 to 23 months) and 21 days (range: 17 to 24 months) in the nCRT and Nimo-nCRT group, respectively. The toxicities are listed in Table 2. Grade 3-4 radiation pneumonitis was not observed, only one patient in the nCRT group developed Grade 2 radiation pneumonitis by week 2 after treatment. Among the patients in the Nimo-nCRT group, one (5.6\%) experienced grade 2 acneiform rash, and four (22.2\%) had grade 1 rash. Hypomagnesemia was noted in one patient $(5.6 \%) 4$ weeks after the initiation of treatment. The allergic reaction to nimotuzumab was not observed.

Six patients $(6.2 \%)$ in the $\mathrm{nCT}$ group, seven $(8.8 \%)$ in the nCRT group and one (5.6\%) in the Nimo-nCRT group required chemotherapy dose reductions, primarily for neutropenia. Radiotherapy needed to be delayed for six patients $(7.5 \%)$ in the nCRT group and one $(5.6 \%)$ in the Nimo-nCRT group, and the treatment interruptions were ranged from 3 to 5 days.

\section{Surgery}

After completion of neoadjuvant therapy, restaging evaluation and the feasibility assessment of performing surgery were conducted by the multidisciplinary team.

Sixteen patients in the $\mathrm{nCT}$ group did not undergo esophagectomy, 4 patients developed distant metastases; 11 were deemed surgically unresectable because 10 patients showed stable diseases and 1 had primary tumor progression; one patient refused to undergo surgery. Seventy-five patients $(92.6 \%)$ underwent an R0 surgical resection of their primary tumor in the 81 patients who proceeded to surgery. The median duration of admission for surgery was 11.5 days (range, 7-58 days), with twelve patients hospitalized for more than 14 days.

Among those in the nCRT group, 74 patients proceeded to surgery and $71(95.9 \%)$ successfully underwent an R0 resection. One patient did not undergo surgery because of progressive disease and four were deemed surgically unresectable, and one patient refused to receive esophagectomy. The median length of hospital stay in those undergoing surgery was 12 days (range 8 to 62 days). 
Table 1: Baseline characteristics at enrollment by treatment group

\begin{tabular}{|c|c|c|c|c|c|c|}
\hline \multirow{2}{*}{ Characteristic } & \multicolumn{2}{|c|}{ nCT $(n=97)$} & \multicolumn{2}{|c|}{ nCRT $(n=80)$} & \multicolumn{2}{|c|}{ Nimo-nCRT $(n=18)$} \\
\hline & No. & $\%$ & No. & $\%$ & No & $\%$ \\
\hline \multicolumn{7}{|l|}{ Gender } \\
\hline Male & 77 & 79.4 & 61 & 76.2 & 14 & 77.8 \\
\hline Female & 20 & 20.6 & 19 & 23.8 & 4 & 22.2 \\
\hline \multicolumn{7}{|l|}{ Age, years } \\
\hline Median & \multicolumn{2}{|c|}{58} & \multicolumn{2}{|c|}{59} & \multicolumn{2}{|c|}{61} \\
\hline Range & \multicolumn{2}{|c|}{$33-71$} & \multicolumn{2}{|c|}{$31-74$} & \multicolumn{2}{|c|}{$46-71$} \\
\hline \multicolumn{7}{|l|}{ Performance status } \\
\hline ECOG 0 & 47 & 48.5 & 37 & 46.2 & 8 & 44.4 \\
\hline ECOG 1 & 50 & 51.5 & 43 & 53.8 & 10 & 55.6 \\
\hline \multicolumn{7}{|l|}{ Tumor location } \\
\hline Upper thoracic & 20 & 20.6 & 29 & 36.2 & 6 & 33.3 \\
\hline Middle thoracic & 70 & 72.2 & 46 & 57.5 & 11 & 61.1 \\
\hline Lower thoracic & 7 & 7.2 & 5 & 6.3 & 1 & 5.6 \\
\hline \multicolumn{7}{|l|}{ Primary tumor size } \\
\hline$\leq 5 \mathrm{~cm}$ & 41 & 42.3 & 29 & 36.2 & 7 & 38.9 \\
\hline$>5 \mathrm{~cm}$ & 56 & 57.7 & 51 & 63.8 & 11 & 61.1 \\
\hline \multicolumn{7}{|l|}{ Clinical stage } \\
\hline IIA & 23 & 23.7 & 19 & 23.8 & 4 & 22.2 \\
\hline IIB & 7 & 7.2 & 2 & 2.5 & 1 & 5.6 \\
\hline IIIA & 35 & 36.1 & 30 & 37.5 & 6 & 33.3 \\
\hline IIIB & 1 & 1.0 & 1 & 1.2 & 0 & 0 \\
\hline IIIC & 31 & 32.0 & 28 & 35.0 & 7 & 38.9 \\
\hline
\end{tabular}

Abbreviations: nCT, neoadjuvant chemotherapy; nCRT, neoadjuvant chemoradiotherapy; Nimo-nCRT, nimotuzumab plus neoadjuvant chemoradiotherapy; ECOG: Eastern Cooperative Oncology Group.

Among the 18 patients in the Nimo-nCRT group, 17 underwent surgery and $\mathrm{R} 0$ resections were achieved in all of them. One did not undergo surgery because of patient refusal. The median duration of admission for surgery was 12 days (range, 7-60 days).

Surgical complications were noted in $32.1 \%$ of patients in the nCT group, $36.5 \%$ in the nCRT group, and $35.3 \%$ in the Nimo-nCRT group, respectively (Figure 1). Infections were the predominant complication of surgery and occurred in about $10 \%$ of the patients in the three groups. One patient in the $\mathrm{nCT}$ group died within 30 days after surgery because of mediastinal abscese, and one patient in the nCRT group died from respiratory failure on the 52nd day of hospitalization. Other major complications including anastomotic leakage, anastomotic stricture, hoarseness, and arrhythmia were comparable among the three groups (Table 3).

\section{Efficacy}

Pathological findings showed that a pCR was achieved in $41.2 \%(7 / 17)$ of the patients in the NimonCRT group, versus $32.4 \%$ (24/74) in the nCRT group and
$14.8 \%(12 / 81)$ in the nCT group $(P=0.000)$. Nimo-nCRT was also associated with a significant increased incidence of ypT0 $(P=0.001)$, ypN0 $(P=0.043)$ compared to nCRT and nCT group. Post-operative pathologic staging determined that $88.2 \%$ of the patients were downstaged following Nimo-nCRT, versus $82.4 \%$ following nCRT and $67.9 \%$ following $\mathrm{nCT}$, a significant difference was found among the three groups $(P=0.000)$. Of patients resected in the Nimo-nCRT group, $29.4 \%$ had metastatic lymph-nodes, versus $21.6 \%$ in the nCRT group and $35.8 \%$ in the nCT group $(P=0.093$; nCRT vs. nCT, $P=0.043)$, as shown in Figure 2.

\section{DISCUSSION}

Neoadjuvant chemotherapy and chemoradiotherapy have convincingly been demonstrated to improve long-term outcomes for patients with locally advanced resectable esophageal cancer, which of the two neoadjuvant therapy types being more beneficial continues to be debated. In 2009, Stahl et al. [14] launched a randomised phase III trial comparing nCRT with nCT; but the study ended prematurely because of slow accrual. 
Table 2: Grade 3 to 4 toxicities associated with the neoadjuvant regimen

\begin{tabular}{|c|c|c|c|c|c|c|}
\hline \multirow{2}{*}{ Adverse event } & \multicolumn{2}{|c|}{$\mathrm{nCT}(n=97)$} & \multicolumn{2}{|c|}{$\operatorname{nCRT}(n=80)$} & \multicolumn{2}{|c|}{ Nimo-nCRT $(n=18)$} \\
\hline & Grade 3 & Grade 4 & Grade 3 & Grade 4 & Grade 3 & Grade 4 \\
\hline \multicolumn{7}{|l|}{ Hematologic } \\
\hline Neutropenia & $19(19.6 \%)$ & $5(5.2 \%)$ & $19(23.8 \%)$ & $6(7.5 \%)$ & $4(22.2 \%)$ & $1(5.6 \%)$ \\
\hline Febrile neutropenia & $5(5.2 \%)$ & $2(2.1 \%)$ & $4(5.0 \%)$ & $2(2.5 \%)$ & $1(5.6 \%)$ & 0 \\
\hline Thrombocytopenia & $4(4.1 \%)$ & 0 & $3(3.8 \%)$ & 0 & $1(5.6 \%)$ & 0 \\
\hline \multicolumn{7}{|l|}{ Nonhematologic } \\
\hline Esophagitis & $3(3.1 \%)$ & 0 & $13(16.3 \%)$ & 0 & $2(11.1 \%)$ & 0 \\
\hline Fatigue & $8(8.2 \%)$ & 0 & $7(8.8 \%)$ & 0 & $1(5.6 \%)$ & 0 \\
\hline Anorexia & $12(12.4 \%)$ & $2(2.1 \%)$ & $9(11.3 \%)$ & $1(1.3 \%)$ & $2(11.1 \%)$ & 0 \\
\hline Constipation & $10(10.3 \%)$ & 0 & $7(8.8 \%)$ & 0 & $1(5.6 \%)$ & 0 \\
\hline Diarrhea & $3(3.1 \%)$ & 0 & $2(2.5 \%)$ & 0 & 0 & 0 \\
\hline Nausea & $22(22.7 \%)$ & 0 & $18(22.5 \%)$ & 0 & $4(22.2 \%)$ & 0 \\
\hline Vomiting & $10(10.3 \%)$ & 0 & $8(10.0 \%)$ & 0 & $1(5.6 \%)$ & 0 \\
\hline Weight loss & $2(2.1 \%)$ & 0 & $2(2.5 \%)$ & 0 & $1(5.6 \%)$ & 0 \\
\hline
\end{tabular}

Abbreviations: nCT, neoadjuvant chemotherapy; nCRT, neoadjuvant chemoradiotherapy; Nimo-nCRT, nimotuzumab plus neoadjuvant chemoradiotherapy.

One hundred twenty-five patients were randomised, and of whom data of 119 patients were analysed. The $\mathrm{R} 0$ resection rate was considerably higher with nCRT $(88 \%)$ than $\mathrm{nCT}$
(79\%); pCR was significantly higher with nCRT (15.6 vs. $2 \%, P=0.03)$ as was the percentage of tumor-free lymph nodes (ypN0, 64.4\%vs. 37.7\%, $P=0.01$ ). Moreover, nCRT

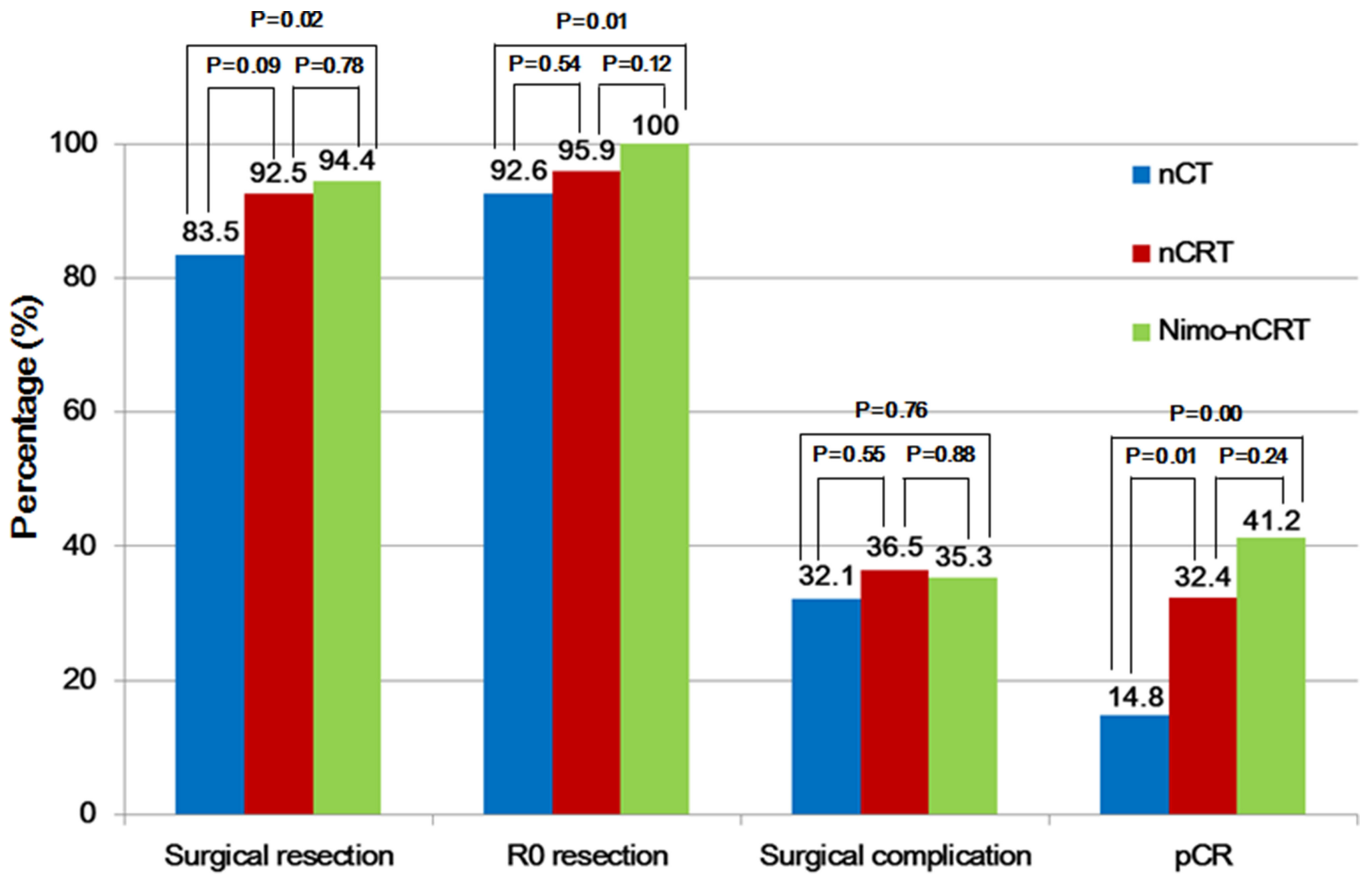

Figure 1: Outcomes related to surgery in the three groups. nCT, neoadjuvant chemotherapy; nCRT, neoadjuvant chemoradiotherapy; Nimo-nCRT, nimotuzumab plus neoadjuvant chemoradiotherapy; pCR, pathologic complete response. 
Table 3: Postoperative complications

\begin{tabular}{lcccccc}
\hline \multirow{2}{*}{ Complication } & \multicolumn{2}{c}{ nCT $(\boldsymbol{n}=\mathbf{8 1})$} & \multicolumn{2}{c}{ nCRT $(\boldsymbol{n}=\mathbf{7 4})$} & \multicolumn{2}{c}{ Nimo-nCRT $(\boldsymbol{n}=\mathbf{1 7})$} \\
\cline { 2 - 7 } & No & $\mathbf{\%}$ & No. & $\mathbf{\%}$ & No. & \% \\
\hline Postoperative infection & 9 & 11.1 & 8 & 10.8 & 2 & 11.8 \\
Anastomotic leakage & 4 & 4.9 & 4 & 5.4 & 1 & 5.9 \\
Mediastinal abscese & 2 & 2.5 & 1 & 1.4 & - & - \\
Anastomotic stricture & 4 & 4.9 & 5 & 6.8 & 1 & 5.9 \\
Pleural effusion & 1 & 1.2 & 2 & 2.7 & - & - \\
Hoarseness & 3 & 3.7 & 3 & 4.1 & 1 & 5.9 \\
Arrhythmia & 3 & 3.7 & 4 & 5.4 & 1 & 5.9 \\
\hline
\end{tabular}

Abbreviations: nCT, neoadjuvant chemotherapy; nCRT, neoadjuvant chemoradiotherapy; Nimo-nCRT, nimotuzumab plus neoadjuvant chemoradiotherapy.

demonstrated a positive trend toward improved 3-year overall survival over $\mathrm{nCT}(47.4$ vs. $27.7 \%)$, though that was not statistically significant $(P=0.07)$. Klevebro et al. [7] compared the effects of nCT with those of nCRT in 181 esophageal cancer patients. The patients in the nCRT arm received three cycles of platin/5-fluorouracil and $40 \mathrm{~Gy}$ of concomitant radiotherapy. The addition of radiotherapy to $\mathrm{nCT}$ resulted in better clinical outcomes, the $\mathrm{R} 0$ resection rate was $74 \%$ after $\mathrm{nCT}$ and $87 \%$ after nCRT $(P=0.04)$, pCR was much higher with nCRT (28 vs.
$9 \%, P=0.002$ ), and lymph-node metastases were reported in $62 \%$ in the nCT group and $35 \%$ in the nCRT group $(P=0.001)$; moreover, survival analysis according to tumor histological type showed a trend towards improved survival among patients with squamous cell carcinoma receiving nCRT.

Studies have demonstrated that paclitaxel is an active agent against esophageal cancer, the single agent activity reached approximately $30 \%$, and it has synergistic anticancer activity in combination with

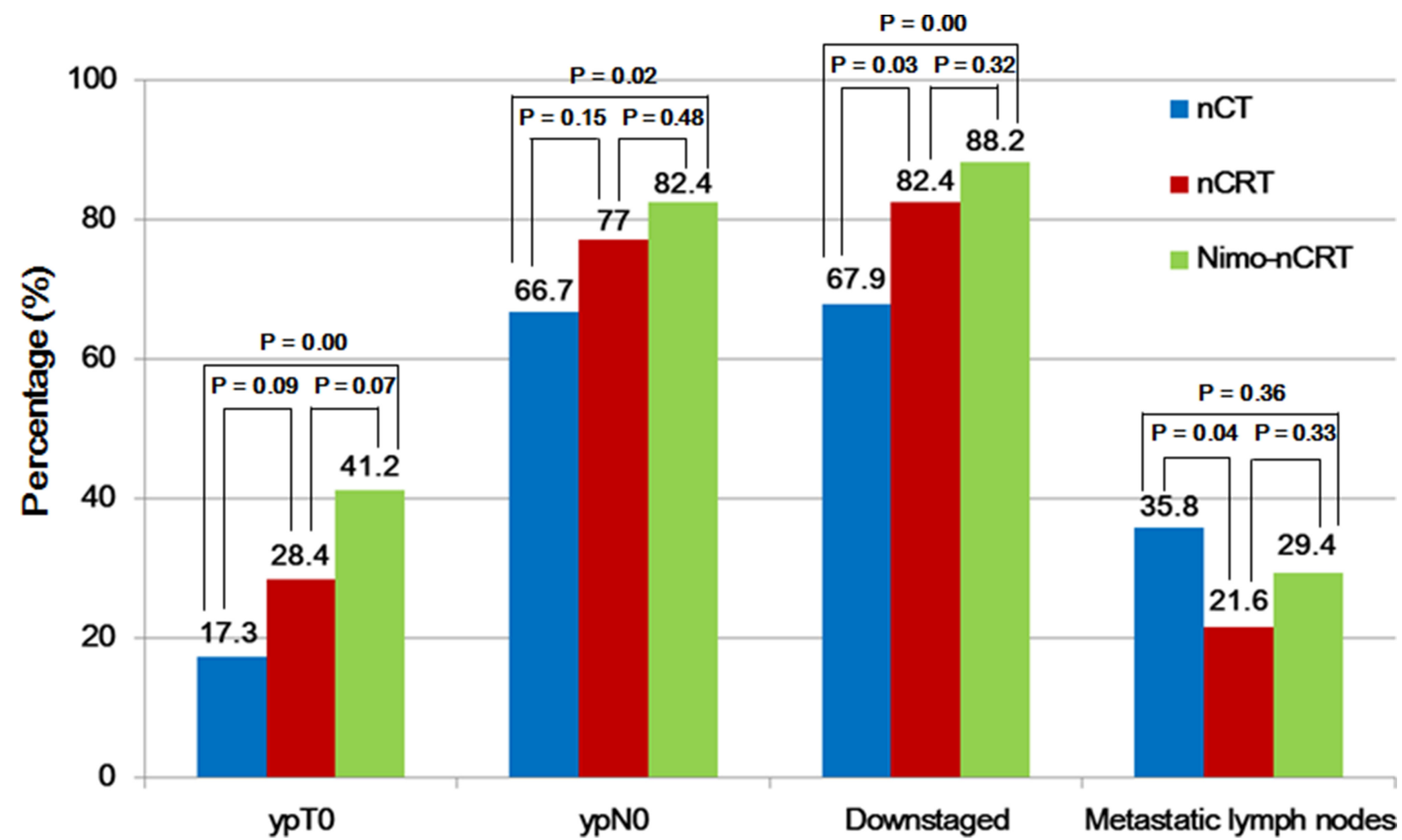

Figure 2: Pathological findings following the three neoadjuvant therapy regimens. nCT, neoadjuvant chemotherapy; $\mathrm{nCRT}$, neoadjuvant chemoradiotherapy; Nimo-nCRT, nimotuzumab plus neoadjuvant chemoradiotherapy; ypT0, pathologic T0 after neoadjuvant therapy; ypN0, pathologic N0 after neoadjuvant therapy. 
Table 4: Comparison of studies of chemoradiotherapy regimens with anti-EGFR antibodies

\begin{tabular}{|c|c|c|c|c|c|}
\hline Year & Study & Phase & Regimen & $\begin{array}{c}\text { No. of patients } \\
\text { (adenocarcinoma: } \\
\text { SCC) }\end{array}$ & ITT pCR \\
\hline 2008 & Safran, et al. [21] & II & $\begin{array}{l}\text { cetuximab, carboplatin, paclitax, and RT } \\
(50.4 \mathrm{~Gy} / 28 \mathrm{f})\end{array}$ & $60(48: 12)$ & $25 \%$ \\
\hline 2010 & Kleinberg, et al. [22] & II & $\begin{array}{l}\text { cetuximab, oxaliplatin, 5-FU, and RT } \\
\text { (45 Gy/25f) }\end{array}$ & $22(22: 0)$ & $32 \%$ \\
\hline 2011 & De Vita F, et al. [23] & II & $\begin{array}{l}\text { cetuximab, oxaliplatin, 5-FU, LV, and RT } \\
\text { (50.4 Gy/28f) }\end{array}$ & $41(13: 28)$ & $27 \%$ \\
\hline 2011 & Ruhstaller, et al. [24] & $\mathrm{IB} / \mathrm{II}$ & $\begin{array}{l}\text { cetuximab, cisplatin, docetaxel, and RT } \\
\text { ( } 45 \mathrm{~Gy} / 25 \mathrm{f})\end{array}$ & $28(15: 13)$ & $32 \%$ \\
\hline 2013 & Lee, et al. [25] & II & $\begin{array}{l}\text { cetuximab, cisplatin, irinotecan, and RT } \\
(50.4 \mathrm{~Gy} / 28 \mathrm{f})\end{array}$ & $19(16: 3)$ & $16 \%$ \\
\hline 2016 & Lledo, et al. [26] & II & $\begin{array}{l}\text { cetuximab, oxaliplatin, } 5-\mathrm{FU}, \mathrm{LV} \text {, and RT } \\
\text { (50.4 Gy/30f) }\end{array}$ & $79(26: 53)$ & NR \\
\hline 2017 & This study & II & $\begin{array}{l}\text { nimotuzumab, cisplatin, paclitax, and RT } \\
\text { (40 Gy/20f) }\end{array}$ & $18(0: 18)$ & $41 \%$ \\
\hline
\end{tabular}

Abbreviations: EGFR, epidermal growth factor receptor; 5-FU, 5-fluorouracil; LV, leucovorin; RT, radiation therapy; SCC, squamous cell carcinoma; ITT, intention to treat; pCR, pathologic complete response; NR, not reported.

cisplatin $[15,16]$. Paclitaxel also acts as a radiosensitizer because it causes $\mathrm{G} 2 / \mathrm{M}$ phase cell cycle arrest, the most radiosensitive phase of the cell cycle [17]. The current practice of nCRT in many Western countries is utilizing the CROSS trial regimen with chemotherapy by weekly paclitaxel and carboplatin, and concomitant radiotherapy (41.4 Gy/1.8 Gy per fraction). In that trail, $94 \%$ of the patients underwent surgery, the rates of R 0 resection, pCR, and lymph node metastases were $92 \%, 29 \%$, and $31 \%$, respectively $[2,3]$. The higher rate of $\mathrm{pCR}$ and longer overall survival among patients with squamous cell carcinoma were noted compared with those with adenocarcinoma, thus esophageal squamous cell carcinoma patients can benefited more from nCRT. The most recent randomised trial (NEOCTRE5010) [18] used nCRT consisting of 2 cycles of cisplatin and vinorelbine with concurrent radiotherapy (40 Gy in 20 fractions, five fractions per week). That trial recruited 451 patients of whom 224 were randomly allocated to the nCRT group, and 227 to the surgery alone group, nCRT increased R0 resection rate from $91.2 \%$ to $98.4 \%(P=0.002)$ and a $43.2 \% \mathrm{pCR}$ rate was achieved.

Neoadjuvant chemoradiotherapy have been proven to prolong the overall survival in patients with resectable thoracic esophageal cancer, but the optimal radiation dose is uncertain, specifically whether low dose CRT is as effective as higher doses. Buckstein and colleagues [19] investigated the neoadjuvant radiation dose and short- and long-term outcomes in the National Cancer Database, 7325 patients with esophageal cancer receiving neoadjuvant CRT followed by curative surgery were identified and four radiation dose levels (40-41.4
Gy, 45 Gy, 50.4 Gy, and 54 Gy) were assessed. After a median follow-up of 26.3 months, radiation dose level was not associated with differences in $\mathrm{pCR}(P=0.21)$ or overall survival $(P=0.39)$. The present study used nCRT consisting of two cycles of paclitaxel and cisplatin with concomitant radiotherapy to dose of $40 \mathrm{~Gy}$ in 20 fractions, $93 \%$ of the patients proceeded to surgery, the rates of R0 resection, pCR, and tumor-free lymph nodes were $96 \%$, $32 \%$, and $78 \%$, respectively. We could conclude from the studies described above, that excellent outcomes can be achieved with low dose radiation and chemotherapy with paclitaxel and cisplatin for neoadjuvant therapy of esophageal cancer.

Anti-EGFR antibodies nimotuzumab and cetuximab inhibit ligand binding to the receptor, thereby stabilize the inactive state of EGFR, nimotuzumab inhibits EGFstimulated, and ligand-independent signaling in EGFRoverexpressing cells [20]. No data was reported about adding nimotuzumab to concurrent chemoradiotherapy administered preoperatively in patients with locally advanced esophageal cancer, however, several chemoradiotherapy combinations with cetuximab have been reported (Table 4) [21-26]. Kleinberg and colleagues [22] used neoadjuvant oxaliplatin plus fluorouracil and radiotherapy (45 Gy in 25 fractions) in combination with cetuximab, followed by esophagectomy and adjuvant cetuximab and docetaxel, and observed a $32 \%$ pCR rate. However, a total of seven deaths occurred during the study's period because of treatment toxicities and postoperative complications. De Vita F and colleagues [23] treated 41 patients with a regimen consisting of 2 months of cetuximab plus FOLFOX-4 followed by 
6 weekly radiotherapy (50.4 Gy in 28 fractions) plus cetuximab. Eight patients achieved pCR among 30 patients who underwent surgery, and the pCR rate was $27 \%$. The most common grade $3 / 4$ toxicity was neutropenia (30\%) and skin rash (30\%).

Study from Lee et al. [25] and the S0414 trial [27] investigated the toxicity and efficacy of cetuximab in combination with irinotecan, cisplatin, and radiotherapy in patients with locally advanced esophageal cancer. They noted substantial toxicity of high frequency of grade 3 or 4 neutropeniain and grade 3 or 4 diarrhea, but the therapeutic efficacy was low.

Combining cetuximab with a taxane, platinum analog and radiotherapy were well tolerated and had achieved promising results for adjuvant treatment of esophageal cancer. Safran and colleagues [21] treated 60 patients using cetuximab in combination with paclitaxel, carboplatin, and radiotherapy (50.4 Gy in 28 fractions). A $27 \%$ pCR rate was achieved in patients who subsequently underwent esophagectomy. The most common grade 3-4 toxicities were rash $(25 \%)$, dehydration (16\%), esophagitis (16\%), and neutropenia (14\%). Ruhstaller et al. [24] investigated cetuximab added to docetaxel, cisplatin, and radiotherapy (45 Gy in 25 fractions) in 28 esophageal cancer patients who were candidates for potentially curative esophagectomy, $32 \%$ of the patients achieved a pCR, and an additional $36 \%$ of those had microscopic residual disease. Though these trials presented promising results, the RTOG 0436 trial [28] and the phase III trial of SCOPE1 [29] showed that the addition of cetuximab to standard chemoradiotherapy regimen failed to improve progression-free survival and overall survival irrespective of tumor histology for patients with esophageal cancer suitable for definitive CRT.

Each modality can have adverse effects when esophageal cancer patients are subjected to chemotherapy, biotherapy, radiation, and surgery, safety is thus a major concern of trimodal treatment approach. The studies above demonstrated that the addition of cetuximab increased treatment-related toxicities, decreased the delivery of all components of standard chemoradiotherapy, and might negatively affect treatment outcomes. However, our study showed that the addition of nimotuzumab to neoadjuvant chemoradiotherapy including paclitaxel and cisplatin was safe, no allergic reaction was noted, and skin toxicity was mild. The regimen did not jeopardize the treatment process, and all 17 patients who underwent surgery achieved an R0 resection, the risk of surgical complications was not increased. The rates of pCR and downstaging in patients undergoing Nimo-nCRT were significantly higher than that in those receiving $\mathrm{nCRT}$ and $\mathrm{nCT}$.

Our study suggests that adding nimotuzumab to nCRT is safe and may facilitate complete resection and increase the $\mathrm{pCR}$ rate for locally advanced esophageal squamous cell carcinoma, the long-term survival and late toxicities are under investigation. The weaknesses of this study are as following: it involves a single-institution experience and the sample size was small, efficacy results should be interpreted with caution; it is a retrospective analysis and the patients, baseline characteristics were not well balance; the biological markers were not evaluated for prognosis. However, our analysis has the following strengths: it focuses on a specific histologic subtype rather than grouping adenocarcinoma and squamous cell carcinoma; it demonstrates that chemoradiation at a dose of $40 \mathrm{~Gy}$ using modern radiotherapy technique for neoadjuvant treatment of esophageal cancer is an effective approach.

\section{MATERIALS AND METHODS}

\section{Patients}

Eligible patients had pathologically proven stage II-III thoracic esophageal squamous cell carcinoma (as defined by the American Joint Committee on Cancer [30]). Other eligibility criteria were as follows: aged 18 to 75 years old; Eastern Cooperative Oncology Group (ECOG) performance status score of 0 or 1 . Required laboratory parameters for inclusion were hemoglobin $\geq 100 \mathrm{~g} / \mathrm{L}$, absolute neutrophil count $(\mathrm{ANC}) \geq 1.5 \times 10^{\%} / \mathrm{L}$, platelets $\geq 100 \times 10^{9} / \mathrm{L}, \mathrm{ALT}$ or AST $<2.5$ times the upper limit of normal (ULN), bilirubin $\leq 1.5$ times the ULN, and serum creatinine $\leq 1.5 \mathrm{~g} / \mathrm{L}$. These participants had no other active malignancy or significant uncontrolled comorbidity, and no prior systemic chemotherapy or chest irradiation.

All patients underwent staging studies including endoscopic ultrasonography (EUS) and EUS guided biopsy; esophagram, neck-to-abdomen computed tomography (CT) scan; or positron emission tomography (PET)/CT scanning, and bronchoscopy if airway infiltration was suspected.

\section{Chemotherapy}

The chemotherapy regimen were administered as follows: paclitaxel given by a 3-hour infusion at a dose of $135 \mathrm{mg} / \mathrm{m}^{2}$ on day 1 , followed by cisplatin at a dose of $25 \mathrm{mg} / \mathrm{m}^{2} /$ day on days $1-3$. Dexamethasone $20 \mathrm{mg}$, ranitidine $50 \mathrm{mg}$ and diphenhydramine $50 \mathrm{mg}$ were given intravenously 30 minutes before infusion of paclitaxel. Participants underwent electrocardiogram monitoring for 5 hours after the beginning of the treatment. The chemotherapy was repeated every 3 weeks with two cycles.

\section{Chemoradiotherapy}

The same chemotherapy regimen was administered, and radiotherapy was initiated on the 1st day of the 1st cycle of chemotherapy. Three-dimensional (3D) conformal 
radiation therapy of 40 Gy (20 fractions of $2.0 \mathrm{~Gy}$ ) was given once daily for 5 days per week using a highenergy linear accelerator $(\geq 6 \mathrm{MV})$. Information from the esophagram, EUS, and CT scan were studied in detail by radiologist and radiation oncologist before delineation of target tumor volume. The gross tumor volume (GTV) included the primary tumor and involved lymph nodes. The clinical target volume (CTV) was created by adding $2 \mathrm{~cm}$ radially and $4 \mathrm{~cm}$ cranially and caudally beyond the GTV. The CTV included the medial supraclavicular fossa in patients with tumor located in the upper thoracic esophagus, and CTV also included the celiac nodal region if the primary tumor occurred in the distal third of the thoracic esophagus. The planning target volume (PTV) was generated using an isotropic three-dimensional expansion of the CTV to $6 \mathrm{~mm}$. Heterogeneity corrections were performed to assure that at least $95 \%$ of the CTV received the prescribed dose.

\section{Nimotuzumab combined with chemoradiotherapy}

The participants in nimotuzumab plus chemoradiotherapy (Nimo-nCRT) group were administrated nimotuzumab by $30 \mathrm{mins}$ I.V. infusion $200 \mathrm{mg}$ weekly for 5 weeks and the same chemoradiotherapy regimen.

\section{Surgery}

Restaging evaluation was performed 4-6 weeks after completion of neoadjuvant therapy, when a neck-to-abdomen CT scan showed no evidence of distant metastasis or inoperable disease, radical esophagectomy with three field lymphadenectomy would be conducted essentially at the sixth week after neoadjuvant therapy.

\section{Assessments and dose modifications}

Toxicity evaluations were measured according to the National Cancer Institute's Common Toxicity Criteria (Version 1.0) and the RTOG Radiation Morbidity Scoring Criteria. Routine blood tests were carried out once every week, and biochemical liver and kidney functions tests once every two weeks. If a patient experienced allergic reaction to nimotuzumab or paclitaxel, the patients was taken off study. Cisplatin was held for serum creatinine level exceeding $2.0 \mathrm{mg} / \mathrm{dL}$, grade 3-4 ototoxicity, and grade 3-4 neuropathy, and the dose was reduced by half if the creatinine level ranged from 1.6 to $2.0 \mathrm{mg} / \mathrm{dL}$. Paclitaxel was held and subsequently dose reduced by $20 \%$ for an ANC $<1000 / \mathrm{mm}^{3}$, or platelets $<75,000 / \mathrm{mm}^{3}$; for febrile neutropenia or bleeding complications. Radiation was temporarily stopped for any of the following: ANC < $1000 / \mathrm{mm}^{3}$, platelets $<50,000 / \mathrm{mm}^{3}$, grade 3 esophagitis/ mucositis, or any grade 4 toxicity. No dose modifications for radiotherapy were allowed.

\section{Statistical methods and follow-up}

Primarily, the pathologic complete response (pCR) rate, tolerability and postoperative complications of the three regimens were compared. A pCR was defined as the absence of cancer cells in the pathologic examination of surgical specimen, the analysis defined tumor downstaging as a decrease in the stage detected on pathological staging after esophagectomy compared to the stage determined in the pretreatment staging workup. An $\mathrm{R} 0$ resection is defined as complete tumor excision with negative histological margins. Pearson's Chisquared test for categorical variables and Student's $t$-test for continuous variables were used to compare patients' baseline characteristics and outcomes between the groups. All $P$ values are two-sided and $P$-values of less than 0.05 were considered to indicate statistical significance. All statistical analyses were performed using SPSS software (version 20.0.1; SPSS Inc., Chicago, IL, USA).

Physical examination, evaluation of ECOG PS, barium swallow, cervical/thoracic/abdominal CT were performed 1 month after the completion of all the therapy. These tests were subsequently performed every 3 months for the first year and every 4 months thereafter. Endoscopic examination was performed if there were new symptoms suggestive of local recurrence.

\section{Author contributions}

1. Conception and design: Yongshun Chen; 2. Provision of study materials: Yongshun Chen, Xiaoyuan Wu, Chunyu He; 3. Data collection: Daxuan Hao, Xinyu Cheng, Lei Zhang, Shaobo Ke, Wei Shi; 4. Data analysis and interpretation: Yongshun Chen, Daxuan Hao, Yougai Zhang; 5. Manuscript writing: Yongshun Chen.

\section{CONFLICTS OF INTEREST}

No conflicts of interest was declared.

\section{FUNDING}

This work was supported by the National Natural Science Foundation of China (No. U1604175). No benefits in any form have been or will be received from a commercial party related to the subject of this work.

\section{REFERENCES}

1. Allum WH, Stenning SP, Bancewicz J, Clark PI, Langley RE. Long-term results of a randomized trial of surgery with or without preoperative chemotherapy in esophageal cancer. J Clin Oncol. 2009; 27:5062-5067. https://doi.org/10.1200/ JCO.2009.22.2083. [PubMed]

2. Van Hagen P, Hulshof MC, van Lanschot JJ, Steyerberg EW, van Berge Henegouwen MI, Wijnhoven BP, Richel 
DJ, Nieuwenhuijzen GA, Hospers GA, Bonenkamp JJ, Cuesta MA, Blaisse RJ, Busch OR, et al. Preoperative chemoradiotherapy for esophageal or junctional cancer. $\mathrm{N}$ Engl J Med. 2012; 366:2074-2084. https://doi.org/10.1056/ NEJMoa1112088. [PubMed]

3. Shapiro J, van Lanschot JJ, Hulshof MC, van Hagen P, van Berge Henegouwen MI, Wijnhoven BP, van Laarhoven HW, Nieuwenhuijzen GA, Hospers GA, Bonenkamp JJ, Cuesta MA, Blaisse RJ, Busch OR, et al. Neoadjuvant chemoradiotherapy plus surgery versus surgery alone for oesophageal or junctional cancer (CROSS): long term results of a randomised controlled trial. Lancet Oncol. 2015; 16:1090-1098. https://doi.org/10.1016/S14702045(15)00040-6. [PubMed]

4. Hulshoff JB, Faiz Z, Karrenbeld A, Kats-Ugurlu G, Burgerhof JGM, Smit JK, Plukker JT. Prognostic value of the circumferential resection margin in esophageal cancer patients after neoadjuvant chemoradiotherapy. Ann Surg Oncol. 2015; 22:S1301-S1309. https://doi.org/10.1245/ s10434-015-4827-2. [uuMed]

5. Donahue JM, Nichols FC, Li Z, Schomas DA, Allen MS, Cassivi SD, Jatoi A, Miller RC, Wigle DA, Shen KR, Deschamps C. Complete pathologic response after neoadjuvant chemoradiotherapy for esophageal cancer is associated with enhanced survival. Ann Thorac Surg. 2009; 87:392-399. https://doi.org/10.1016/j.athoracsur.2008.11.001. [PubMed]

6. Taylor MD, Lapar DJ, Davis JP, Isbell JM, Kozower $\mathrm{BD}$, Lau CL, Jones DR. Induction chemoradiotherapy and surgery for esophageal cancer: survival benefit with downstaging. Ann Thorac Surg. 2013; 96:225-231. https:// doi.org/10.1016/j.athoracsur.2013.01.074. [PubMed]

7. Klevebro F, Alexandersson von Döbeln G, Wang N, Johnsen G, Jacobsen AB, Friesland S, Hatlevoll I, Glenjen NI, Lind P, Tsai JA, Lundell L, Nilsson M. A randomized clinical trial of neoadjuvant chemotherapy versus chemoradiotherapy for cancer of the oesophagus or gastro-oesophageal junction. Ann Oncol. 2016; 27:660-667. https://doi.org/10.1093/ annonc/mdw010. [PubMed]

8. Gibault L, Metges JP, Conan-Charlet V, Lozac'h P, Robaszkiewicz M, Bessaguet C, Lagarde N, Volant A. Diffuse EGFR staining is associated with reduced overall survival in locally advanced oesophageal squamous cell cancer. Br J Cancer. 2005; 93:107-115. https://doi. org/10.1038/sj.bjc.6602625. [PubMed]

9. Wang KL, Wu TT, Choi IS Wang H, Resetkova E, Correa AM, Hofstetter WL, Swisher SG, Ajani JA, Rashid A, Albarracin CT. Expression of epidermal growth factor receptor in esophageal and esophagogastric junction adenocarcinomas: association with poor outcome. Cancer. 2007; 109:658-667. https://doi.org/10.1002/cncr.22445. [PubMed]

10. Ramos-Suzarte M, Lorenzo-Luaces P, Lazo NG, Perez ML, Soriano JL, Gonzalez CE, Hernadez IM, Albuerne YÁ, Moreno BP, Alvarez ES, Callejo IP, Alert J, Martell JA, et al. Treatment of malignant, non-resectable, epithelial origin esophageal tumours with the humanized anti-epidermal growth factor antibody nimotuzumab combined with radiation therapy and chemotherapy. Cancer Biol Ther. 2012; 13:600-605. https://doi.org/10.4161/cbt.19849. [PubMed]

11. Ling Y, Chen J, Tao M, Chu X, Zhang X. A pilot study of nimotuzumab combined with cisplatin and 5-FU in patients with advanced esophageal squamous cell carcinoma. J Thorac Dis. 2011; 4:58-62. https://doi.org/10.3978/j. issn.2072-1439.2011.08.02. [PubMed]

12. Ma NY, Cai XW, Fu XL Li Y, Zhou XY, Wu XH, Hu XC, Fan M, Xiang JQ, Zhang YW, Chen HQ, Lai ST, Jiang GL, Zhao KL. Safety and efficacy of nimotuzumab in combination with radiotherapy for patients with squamous cell carcinoma of the esophagus. Int J Clin Oncol. 2014; 19:297-302. https://doi.org/10.1007/s10147-013-0564-3. [PubMed]

13. Lu M, Wang XC, Shen L, Jia J, Gong J, Li J, Li J, Li Y, Zhang X, Lu Z, Zhou J, Zhang X. Nimotuzumab plus paclitaxel and cisplatin as the first line treatment for advanced esophageal squamous cell cancer: A single centre prospective phase II trial. Cancer Sci. 2016; 107:486-490. https://doi.org/10.1111/cas.12894. [PubMed]

14. Stahl M, Walz MK, Stuschke M, Lehmann N, Meyer HJ, Riera-Knorrenschild J, Langer P, Engenhart-Cabillic R, Bitzer M, Königsrainer A, Budach W, Wilke H. Phase III comparison of preoperative chemotherapy compared with chemoradiotherapy in patients with locally advanced adenocarcinoma of the esophagogastric junction. J Clin Oncol. 2009; 27:851-856. https://doi.org/10.1200/ JCO.2008.17.0506. [PubMed]

15. Ajani JA, Ilson DH, Daugherty K, Pazdur R, Lynch PM, Kelsen DP. Activity of taxol in patients with squamous cell carcinoma and adenocarcinoma of the esophagus. J Natl Cancer Inst. 1994; 86:1086-1091. https://doi.org/10.1093/ jnci/86.14.1086. [PubMed]

16. Kano Y, Akutsu M, Tsunoda S, Mori K, Suzuki K, Adachi $\mathrm{KI}$. In vitro schedule-dependent interaction between paclitaxel and cisplatin in human carcinoma cell lines. Cancer Chemother Pharmacol. 1996; 37:525-530. https:// doi.org/10.1007/s002800050424. [PubMed]

17. Zanelli GD, Quaia M, Robieux I Bujor L, Santarosa M, Favaro D, Spada A, Caffau C, Gobitti C, Trovò MG. Paclitaxel as a radiosensitiser: a proposed schedule of administration based on in vitro data and pharmacokinetic calculations. Eur J Cancer. 1997; 33:486-492. https://doi. org/10.1016/S0959-8049(97)89026-0. [PubMed]

18. Yang H, Fu J, Liu M, Chen Y, Chen Z, Zhu C, Yang H, Fang W, Wang J, Yu Z, Pang Q, Mao W, Zheng X, et al. A phase III clinical trial of neoadjuvant chemoradiotherapy followed by surgery versus surgery alone for locally advanced squamous cell carcinoma of the esophagus. Ann Oncol. 2016; 27:suppl 6. https://doi.org/10.1093/annonc/mdw371.03.

19. Buckstein M, Rhome RM, Ohri N. Neoadjuvant chemoradiation dose and outcomes in esophageal cancers, 
a National Cancer Data Base Study. Int J Radiat Oncol Biol Phys. 2016; 96:S190. https://doi.org/10.1016/j. ijrobp.2016.06.474.

20. Berger C, Krengel U, Stang E, Moreno E, Madshus IH. Nimotuzumab and cetuximab block ligandindependent EGF receptor signaling efficiently at different concentrations. J Immunother. 2011; 34:550-555. https:// doi.org/10.1097/CJI.0b013e31822a5ca6. [PubMed]

21. Safran H, Suntharalingam M, Dipetrillo T, Ng T, Doyle LA, Krasna M, Plette A, Evans D, Wanebo H, Akerman P, Spector J, Kennedy N, Kennedy T. Cetuximab with concurrent chemoradiation for esophagogastric cancer: Assessment of toxicity. Int J Radiat Oncol Biol Phys. 2008; 70:391-395. https://doi.org/10.1016/j.ijrobp.2007.07.2325. [PubMed]

22. Kleinberg LR, Catalano PJ, Gibson MK, Staley CA, Montgomery EA, Song W, Mulcahy MF, Leichman LP, Benson AB. ECOG 2205: A phase II study to measure response rate and toxicity of neo-adjuvant chemoradiotherapy (CRT) (IMRT permitted) with oxaliplatin (O) and infusional 5-fluorouracil (5FU) plus cetuximab (C225) in patients with operable adenocarcinoma of the esophagus: High risk of post-op adult respiratory distress syndrome (ARDS). Int J Radiat Oncol Biol Phys. 2010; 78:S72. https://doi.org/10.1016/j.ijrobp.2010.07.201.

23. De Vita F, Orditura M, Martinelli E, Vecchione L, Innocenti R, Sileni VC, Pinto C, Di Maio M, Farella A, Troiani T, Morgillo F, Napolitano V, Ancona E, et al. A multicenter phase II study of induction chemotherapy with FOLFOX-4 and cetuximab followed by radiation and cetuximab in locally advanced oesophageal cancer. Br J Cancer. 2011; 104:427432. https://doi.org/10.1038/sj.bjc.6606093. [PubMed]

24. Ruhstaller T, Pless M, Dietrich D, Kranzbuehler H, von Moos R, Moosmann P, Montemurro M, Schneider PM, Rauch D, Gautschi O, Mingrone W, Widmer L, Inauen R, et al. Cetuximab in combination with chemoradiotherapy before surgery in patients with resectable, locally advanced esophageal carcinoma: A prospective, multicenter phase
IB/II trial (SAKK75/06). J Clin Oncol. 2011; 29:626-631. https://doi.org/10.1200/JCO.2010.31.9715. [PubMed]

25. Lee MS, Mamon HJ, Hong TS, Choi NC, Fidias PM, Kwak EL, Meyerhardt JA, Ryan DP, Bueno R, Donahue DM. Preoperative cetuximab, irinotecan, cisplatin, and radiation therapy for patients with locally advanced esophageal cancer. The Oncologist. 2013; 18:281-287. https://doi. org/10.1634/theoncologist.2012-0208. [PubMed]

26. Lledo G, Huguet F, Chibaudel B, Di Fiore F, Mineur L, Galais MP, Artru P, Blondin V, Dupuis O, Abdiche MS, Jovenin N, Pozet A, Bonnetain F, et al. Chemoradiotherapy with FOLFOX plus cetuximab in locally advanced oesophageal cancer: The GERCOR phase II trial ERaFOX. Eur J Cancer. 2016; 56:115-121. https://doi.org/10.1016/j. ejca.2015.12.020. [ [pubMed]

27. Tomblyn MB, Goldman BH, Thomas CR Jr, Benedetti JK, Lenz HJ, Mehta V, Beeker T, Gold PJ, Abbruzzese JL, Blanke CD. Cetuximab plus cisplatin, irinotecan, and thoracic radiotherapy as definitive treatment for locally advanced, unresectable esophageal cancer: A phase-II study of the SWOG (S0414). J Thorac Oncol. 2012; 7:906-912. https://doi.org/10.1097/JTO.0b013e31824c7bed. [PubMed]

28. Ilson D, Winter K, Suntharalingham M. RTOG 0436: A phase III trial of cisplatin, paclitaxel and radiation with or without cetuximab in the nonoperative treatment of esophageal cancer. Ann Oncol. 2014; 25(suppl 2):ii106. https://doi.org/10.1093/annonc/mdu193.5.

29. Crosby T, Hurt CN, Falk S, Gollins S, Staffurth J, Ray R, Bridgewater JA, Geh JLI, Cunningham D, Blazeby J, Roy R, Maughan T, Griffiths G, Mukherjee S. Long-term results and recurrence patterns from SCOPE-1: a phase II/III randomised trial of definitive chemoradiotherapy $+/$ - cetuximab in oesophageal cancer. Br J Cancer. 2017; 116:709-716. https:// doi.org/10.1038/bjc.2017.21. [PubMed]

30. Edge SB, Byrd DR, Compton CC, Editors. AJCC Cancer Staging Manual, 7th edition. New York:Springer, 2009: 103-111. 